\title{
BMJ Open Case management in primary care for frequent users of healthcare services with chronic diseases and complex care needs: an implementation and realist evaluation protocol
}

Catherine Hudon, ${ }^{1}$ Maud-Christine Chouinard, ${ }^{2}$ Kris Aubrey-Bassler, ${ }^{3}$ Frederick Burge, ${ }^{4}$ Shelley Doucet, ${ }^{5}$ Vivian R Ramsden, ${ }^{6}$ Magaly Brodeur, ${ }^{1}$ Paula L Bush, ${ }^{7}$ Yves Couturier, ${ }^{8}$ Marie-France Dubois, ${ }^{9}$ Line Guénette, ${ }^{10}$ France Légare, ${ }^{11}$ Paul Morin, ${ }^{8}$ Thomas G Poder, ${ }^{1,12}$ Marie-Ève Poitras, ${ }^{2}$ Pasquale Roberge, ${ }^{1}$ Ruta Valaitis, ${ }^{13}$ Shirley Bighead, ${ }^{14}$ Cameron Campbell, ${ }^{15}$ Martine Couture, ${ }^{16}$ Breanna Davis, ${ }^{6}$ Élaine Deschenes, ${ }^{17}$ Lynn Edwards, ${ }^{18}$ Sarah Gander, ${ }^{19}$ Gilles Gauthier, ${ }^{20}$ Patricia Gauthier, ${ }^{21}$ Richard J Gibson, ${ }^{18}$ Julie Godbout, ${ }^{16}$ Geneviève Landry, ${ }^{22}$ Christine Longjohn, ${ }^{14}$ Norma Rabbitskin, ${ }^{14}$ Denis A Roy, ${ }^{23}$ Judy Roy, ${ }^{24}$ Véronique Sabourin, ${ }^{20}$ Tara Sampalli, ${ }^{18}$ Amanda Saulnier, ${ }^{24}$ Claude Spence, ${ }^{20}$ Jennifer Splane, ${ }^{25}$ Mike Warren, ${ }^{26}$ Joanne Young, ${ }^{27}$ Pierre Pluye ${ }^{7}$

To cite: Hudon C, Chouinard M-C, AubreyBassler K, et al. Case management in primary care for frequent users of healthcare services with chronic diseases and complex care needs: an implementation and realist evaluation protocol. BMJ Open 2018;8:e026433. doi:10.1136/ bmjopen-2018-026433

- Prepublication history for this paper is available online To view these files, please visit the journal online (http://dx.doi org/10.1136/bmjopen-2018026433).

Received 31 August 2018 Revised 17 September 2018 Accepted 19 September 2018

\section{Check for updates}

(C) Author(s) (or their employer(s)) 2018. Re-use permitted under CC BY-NC. No commercial re-use. See rights and permissions. Published by BMJ.

For numbered affiliations see end of article.

\section{Correspondence to}

Professor Catherine Hudon; catherine.hudon@usherbrooke. ca

\section{ABSTRACT}

Introduction Significant evidence in the literature supports case management (CM) as an effective intervention to improve care for patients with complex healthcare needs. However, there is still little evidence about the facilitators and barriers to $\mathrm{CM}$ implementation in primary care setting. The three specific objectives of this study are to: (1) identify the facilitators and barriers of CM implementation in primary care clinics across Canada; (2) explain and understand the relationships between the actors, contextual factors, mechanisms and outcomes of the CM intervention; (3) identify the next steps towards CM spread in primary care across Canada.

Methods and analysis We will conduct a multiple-case embedded mixed methods study. CM will be implemented in 10 primary care clinics in five Canadian provinces. Three different units of analysis will be embedded to obtain an in-depth understanding of each case: the healthcare system (macro level), the CM intervention in the clinics (meso level) and the individual/patient (micro level). For each objective, the following strategy will be performed: (1) an implementation analysis, (2) a realist evaluation and (3) consensus building among stakeholders using the Technique for Research of Information by Animation of a Group of Experts method.

Ethics and dissemination This study, which received ethics approval, will provide innovative knowledge about facilitators and barriers to implementation of CM in different primary care jurisdictions and will explain how and why different mechanisms operate in different contexts to generate different outcomes among frequent users. Consensual and prioritised statements about next steps for spread of $\mathrm{CM}$ in primary care from the
Strengths and limitations of this study

- This multiple-case embedded mixed methods study will provide new knowledge on the implementation of case management (CM) interventions to improve care integration for individuals/patients who frequently use healthcare services.

- The design of this study allows adapting the knowledge acquired on CM to local contexts, which is the first step to implementation.

- The multiprovincial nature of this study will allow to spread the new knowledge generated on CM in primary care settings in different Canadian jurisdictions and will increase generalisability.

- While some challenges are expected with this study, mitigated strategies are nevertheless proposed.

perspectives of all stakeholders will be provided. Our results will offer context-sensitive explanations that can better inform local practices and policies and contribute to improve the health of patients with complex healthcare needs who frequently use healthcare services. Ultimately, this will increase the performance of healthcare systems and specifically mitigate ineffective use and costs.

\section{INTRODUCTION}

In Canada, as in many industrialised countries, ${ }^{12}$ close to $80 \%$ of healthcare costs are attributable to $10 \%$ of the population. ${ }^{34}$ Data reveal that this $10 \%$ segment of the population 
comprises individuals/patients who frequently use hospital services for increasingly complex healthcare needs. Thus, although relatively small, this segment of the population uses a disproportionate amount of available healthcare and social services. Frequent use of emergency departments (EDs) is a good proxy of high use of other healthcare services ${ }^{5-7}$ as it is most commonly accepted in the literature ${ }^{8-12}$ and provides a convenient and easy measure within a pragmatic context, as compared with cost for example. As such, $5 \%$ of ED's patients account for $30 \%-50 \%$ of all visits. ${ }^{813}$ As frequent use is not optimal for individuals/patients ${ }^{14}$ or healthcare systems, ${ }^{15}{ }^{16}$ better upstream care is a modifiable parameter that can effectively prevent it. Indeed, the majority of these individuals/patients who frequently use hospital services have a substantial burden of disease and would be best managed in primary care. ${ }^{17}$

In line with the Agency for Healthcare Research and Quality Multiple Chronic Conditions Research Network, complex healthcare needs can be defined as the gap between an individual's needs and the ability of health services to meet those needs. ${ }^{18}$ Individuals/patients with complex healthcare needs often attempt to fulfil their unmet needs by using excessive health and social services in an uncoordinated way. Requiring a variety of services from various systems (eg, health, social and education) and community networks often leads to difficulties with the integration of care. ${ }^{19}$ This results in negative experiences for individuals/patients, ${ }^{14}$ poorer health outcomes, high mortality rates and considerable costs. ${ }^{19}$

Case management (CM) was reported to be effective for individuals/patients who frequently used healthcare services. ${ }^{10}{ }^{1120}$ By definition, CM is a collaborative approach used to assess, plan, facilitate and coordinate care to meet individual/patient and family healthcare needs, through communication and available resources including all sectors of healthcare (such as community, primary, secondary and tertiary care), as well as sectors outside of the health system (such as social services, housing and so on) with the intent of improving individual and health system outcomes. ${ }^{21}$ Three systematic reviews (including randomised controlled trials, non-randomised controlled trials, interrupted time series and controlled and non-controlled before-and-after studies) concluded that CM was effective for individuals/patients who frequently used healthcare services, particularly on ED use and cost as well as on social and clinical outcomes. ${ }^{81011}$ A scoping review conducted by our team corroborated these findings by revealing that CM could reduce $\mathrm{ED}$ visits and hospitalisations as well as costs. ${ }^{9}$

However, despite the evidence supporting $\mathrm{CM}$ as an effective intervention for individuals/patients that frequently use services, there is still a paucity of evidence about the facilitators and barriers to $\mathrm{CM}$ implementation. ${ }^{10} 22$ Our literature review with thematic analysis of key factors of CM interventions among frequent users of healthcare services outlined that the case finding processes, the selection and training of the case manager, the intensity of the intervention, as well as care integration among all partners were important aspects to consider during CM implementation. ${ }^{22}$

CM has rarely been implemented in primary care in Canada. Therefore, before spreading this intervention in primary care settings in different jurisdictions, stakeholders including individuals/patients/communities need to be engaged in adapting the intervention to their local context. Accordingly, further research is needed to better understand the facilitators and barriers (mechanisms) to CM implementation, as well as the influence of different primary care contexts on outcomes, for example, self-management, quality of life, services integration, services use and costs. 92324

Therefore, the specific objectives of this study are threefold: (1) identify the facilitators and barriers of CM implementation in primary care clinics across Canada; (2) explain and understand the relationships between the actors, contextual factors, mechanisms and outcomes of the CM intervention; and (3) identify the next steps towards CM spread in primary care across Canada.

\section{METHODS AND ANALYSIS}

\section{Study design}

To address these objectives, we will conduct, between September 2018 and August 2022, a multiple-case embedded mixed methods study, which constitutes a valuable design for performing research evaluation inquiries on complex systems in varied and dynamic contexts. ${ }^{2526}$ In addition to allowing an in-depth analysis of each case, this design offers opportunities for comparison between cases. The inclusion of multiple cases capitalises on organisational variation and allows for examination of how contextual factors influence implementation to develop a more informed understanding of change processes. It also allows for observation recursive or singular facilitators and barriers and draws conclusions that could be transferable to other primary care contexts. ${ }^{27}$ Furthermore, mixed methods involve combining qualitative and quantitative methods in complex programme evaluation, primary research and literature review; they are being increasingly used in health sciences; specifically, case studies can use qualitative, quantitative and mixed methods (multiple sources of evidence) to explain one or more cases. ${ }^{28}$

\section{Study location and sampling}

Five Canadian provinces are involved in the study: Saskatchewan, Quebec, Nova Scotia, New Brunswick and Newfoundland and Labrador. Considering that different primary care team models have been implemented throughout Canada, ${ }^{29}{ }^{30}$ the primary care context of each jurisdiction will be taken into account when evaluating implementation and outcomes. ${ }^{30}$

Two primary care clinics per province, where CM has not been previously implemented, will be recruited using a purposeful sampling strategy. ${ }^{31}$ The recruitment 
of the clinics will be conditional to: the manager and team interest in implementing CM and engaging in the research project and availability and interest of a registered nurse or nurse practitioner to develop the role of the case manager. We will thus work with 10 cases (two per province), each case being the intervention implemented in each clinic. It is recommended that $4-10$ cases be considered ${ }^{32}$ in the multiple case study logic of theoretical replication, in which contrasting results are anticipated..$^{25}$ Two clinics per province will facilitate variability within each province. Cases will be selected in order to represent real-world differences ${ }^{33}$ in terms of geographic location, model of practice, diversity of care teams and size based on the opinion of team members in each jurisdiction. Three different units of analysis will be explored to obtain an in-depth understanding of each case: (A) the healthcare system (macro level);

(B) the CM intervention in the clinics (meso level) and (C) the patient including their family and community (micro level).

\section{Objective 1: to identify facilitators and barriers of CM implementation in primary care clinics in Canada}

An implementation analysis will be conducted for identifying facilitators and barriers to, and informing implementation of, CM in primary care in different provinces. ${ }^{34}$ Implementation analysis is very useful with complex interventions that can be influenced by the context within dynamic environments. The case study design is appropriate for implementation analysis of interventions. ${ }^{34}$

\section{Conceptual framework}

Data collection and analysis will rely on the Consolidated Framework for Implementation Research (CFIR) of Damschroder et $a l^{35}$, which is aimed to foster implementation of findings into practice. The CFIR is composed of five major domains: outer setting, inner setting, characteristics of the individuals involved, intervention characteristics and the process of implementation. Four constructs are related to the outer setting (eg, external policies); 12 are related to the inner setting (eg, culture and leadership engagement); five are related to individual characteristics (eg, knowledge and beliefs); eight are related to the intervention (eg, adaptability); and eight are related to process (eg, planning). 'The CFIR provides a practical structure for approaching complex and transient states of constructs in the real world by embracing, consolidating, and unifying key constructs from published implementation theories. ${ }^{35}$

To properly address care integration, the CFIR will be linked to the Valentijn Framework ${ }^{36}$ combining the concepts of primary care and integrated care. In this framework, person-focused care is the guiding principle for achieving integration across the care continuum, that is, system integration (macro level), professional and organisational integration (meso level) as well as clinical integration (micro level).

\section{Preimplementation}

A CM nurse mentor will facilitate 3-day training sessions for all CM nurses and will also lead monthly 1-hour community-of-practice meetings by teleconference to assist with mentoring, collective learning and support. ${ }^{37}$ As recommended by Damschroder et al's CFIR, ${ }^{35}$ team stakeholders will interact with the clinics in their province to codesign the adaptation of the CM intervention to their reality. According to the CFIR, ${ }^{35}$ the core components of the intervention, such as patient assessment, individualised care plan, care coordination and self-management support, ${ }^{38-41}$ will be maintained across all clinics, whereas more peripheral elements will be adaptable, for example, as integration in the context. This adaptability will increase knowledge uptake $\mathrm{e}^{23}$ and promote integration with complementary programmes outside of the clinics, while ensuring that CM is being rigorously evaluated.

\section{Recruitment}

Each clinic will identify 30 patients with the most complex healthcare needs and who, according to their clinical experience of the existing gap between the individual's needs and the ability of health services to meet those needs,${ }^{18}$ could benefit from CM. Inclusion criteria will be: living with at least one chronic condition; frequent ED users as defined by $\geq 4 \mathrm{ED}$ visits in the previous year ${ }^{42} 43$ (which have been recognised as a good proxy of frequent use of other healthcare services ${ }^{5-7}$ ); and a score $\geq 17$ on the INTERMED-Self-Assessment Questionnaire ${ }^{44}$ evaluating complex healthcare needs. Exclusion criteria will be: frail elderly with loss of autonomy; individuals/ patients without a chronic condition or with a prognosis of less than a year; or patients already followed by a case manager in another programme, for example, mental health, senior care and addiction program. Case managers will offer the CM intervention to these individuals/patients over a 12-month period.

\section{Intervention}

The intervention will focus on four main recognised components of $\mathrm{CM}^{38-41}$ : (1) evaluation of patient needs and preferences; (2) codevelopment and maintenance of a patient-centred individualised care plan, with the patient, family and other partners; (3) coordination of health and social services among all partners; and (4) education and self-management support for patients and families. This intervention is congruent with criteria from the Case Management Society of America ${ }^{21}$ and the six standards of practice of the National Case Management Network of Canada $^{45}$ : (1) determining and verifying patient eligibility; (2) assessing patient needs; (3) documenting patient goals and priorities; (4) planning and adjusting services included in individualised service plans, including patient education and self-management support; (5) monitoring patient needs and progress; and (6) supporting transition processes. The intervention also aligns with the six care integration characteristics proposed to consider a patient's experience ${ }^{46}:$ (1) consideration of patient and 
family needs; (2) communication with the patient and between healthcare providers; (3) access to information; (4) involvement in decision making; (5) care planning; and (6) transitions between various professionals.

\section{Data collection}

The mixed method data collection will rely on the five following complementary strategies.

1. Individual semistructured interviews (qualitative data) will be conducted between 6 months and 9 months following initiation of $\mathrm{CM}$ intervention with all case managers, patients/families and clinic managers. Two focus groups per clinic will be also scheduled, enrolling eight primary care providers per group (including physicians, nurses, social workers, pharmacists and others) through purposive sampling. ${ }^{47}$ All interviews and focus groups, conducted using a semistructured interview guide composed of open-ended questions on facilitators and barriers of CM implementation and adapted to each category of stakeholders will be digitally recorded and transcribed verbatim. Interview guides will address the domains and constructs of Damschroder's CFIR ${ }^{35}$ and Valentijn Framework. ${ }^{36}$ Data saturation will not necessarily be reached for each category of stakeholders, but their diversity will allow for a comprehensive representation of each case. ${ }^{48}$

2. Non-participant observation (qualitative data) of CM activities and meetings, for example, patient-case manager, individualised service plan development, team discussions, at each clinic for 30 hours at 6 months will be conducted. Research assistants will collect data by means of an observation grid and field notes. ${ }^{47}$

3. Self-administered and validated questionnaires (quantitative data) with accepted psychometric properties will be administered to all individuals/patients in the presence of the research assistant. At baseline, the following characteristics will be assessed: age; gender; marital status; education; occupation; economic status with family income and patient perception of his or her economic situation; health literacy ${ }^{49}{ }^{50}$; multimorbidity $^{5152}$; care integration ${ }^{53}$; self-management ${ }^{5455}$; and health-related quality of life. ${ }^{56}$ Care integration, self-management and health-related quality of life will be re-evaluated at 12 months.

4. Clinical data on service use during the year of the intervention (quantitative data) will be collected through the patient's electronic medical record: ED visits, overnight stays, primary care and specialist visits. Costs will be measured from a healthcare system perspective, including costs of the CM intervention and of healthcare expenditures. Costs of the intervention will consider nurse training, mentoring and CM implementation. Participant healthcare expenditures, such as ED visits, overnight stays and professional visits, will be calculated using predetermined fees, for example, from the CIHI Patient Cost Database. ${ }^{57}$

5. Intervention fidelity evaluation (quantitative data) will be assessed to determine whether the intervention was delivered as intended. ${ }^{58}$ For this purpose, research assistants will collect data relevant to the delivery of the main components of the CM intervention from the medical records of participants after 6 months and 12 months using a fidelity grid. Similar data on CM intervention fidelity were collected successfully in our previous study. ${ }^{59}$

\section{Data analysis}

Qualitative data analysis: interview-based and observation-based data will be analysed together using a deductive (themes based on the Damschroder et als CFIR and Valentijn Framework) and inductive (themes suggested by the data while not in frameworks) thematic analyses. ${ }^{60}$ Qualitative data will be managed using multisite NVivo V.10 server software (QSR International Pty).

Quantitative data analysis: descriptive statistics will be performed. Intervention fidelity will be represented by the proportion of delivery for each component of the CM intervention. Regression models will be developed to evaluate relationships between contextual elements, that is, intervention fidelity, patients' characteristics and outcomes, using SPSS V.24. An incremental cost-effectiveness/utility ratio ${ }^{61}$ will be calculated, using data collected on costs and quality-adjusted life year (QALY) (ie, short - form six-dimension (SF-6D)), at baseline and 12 months after the CM implementation. Multivariate parametric analyses with bootstrap replications will be conducted along with cost-effectiveness acceptability curves. ${ }^{62}$

Integration of qualitative and quantitative methods: two types of integration will be performed: qualitative and quantitative results will be compared, and qualitative and quantitative data will be merged for each case. ${ }^{28}$ Considering the inherent variety and changing contexts of the study, results of qualitative and quantitative data analyses will be compared, and the comparison will be interpreted using a side-by-side joint comparison table (rather than trying to calculate non-biased quantitative effects ${ }^{63}$ ). Then for each case, qualitative and quantitative data will be merged. ${ }^{25} \mathrm{~A}$ case history will be reported (synthesising merged data), and the 10 case histories will be used to compare cases by means of a descriptive and interpretative matrix (mixed methods matrix), allowing systematic comparisons among cases and analysis units (macro, meso and micro). ${ }^{60}$ Different analytical techniques for case study will be used among which pattern comparison, research of competing explanations and construction of explanations. ${ }^{25}$ Management, data reduction and cross care comparisons will be conducted with NVivo V.10 software using matrix queries. All categories of stakeholders will be invited to participate in key steps of the analysis to ensure meaningful interpretation.

\section{Objective 2: to explain and understand the relationships between actors, contextual factors, mechanisms and outcomes of CM intervention}

A realist evaluation will be conducted according to Pawson and Tilley ${ }^{64}$ Realist evaluation is a theory-driven 
approach for studying complex interventions to explain how and why they are effective, under what conditions and for which groups of patients. It is based on four concepts for explaining and understanding the complex relationships in a given intervention: context, mechanism, outcome and context-mechanism-outcome (CMO) configuration. ${ }^{64-66}$ The multiple-case study is a recognised design for investigating CMO configurations in healthcare research. ${ }^{67-72}$ The realist evaluation will use a multimethod (quantitative and qualitative), theory-driven approach to provide an explanation of why outcomes occur ${ }^{66}$ and will follow three phases: stating an initial programme theory; testing this programme theory; and refining this programme theory.

\section{Stating an initial programme theory}

A proposed initial middle-range programme theory developed in our realist synthesis ${ }^{73}$ of the literature on CM for individuals/patients that frequently use healthcare services in primary care will provide a rigorous basis for the next two phases of data collection (testing and refining the programme theory).

\section{Data collection (testing and refining the programme theory)}

In the next year, same participant sampling and data collection will be repeated in the same clinics identified in objective 1, with a new cohort of patients. However, qualitative data will be used to identify and better understand CMOs. The same quantitative data will be used to measure outcomes, that is, self-management, health-related quality of life, care integration, services use and costs at baseline, 6 months and 12 months for developing CMOs. For qualitative data collection, interview guides and the observation grid will be informed by the initial theory and tailored to the participant groups. Interviews and focus groups will be performed using realist interview techniques. ${ }^{74}$ The theory will be discussed with individuals/patients who will then provide their own experience and vision for collaborative conceptual refinement. The interviewer will play an active role in explaining the contexts and outcomes of interest and in ensuring that participants understand the terminology of the realist evaluation. Participants will be asked to share how they think their experience relates to this theory and to reflect on what may explain the outcomes in their setting. ${ }^{75}$ Data collection will be iterative until reaching saturation. ${ }^{6474}$

\section{Data analysis}

Quantitative data will be analysed, as described above, to inform outcomes. Qualitative data, including interviews, focus groups and observation, will be analysed with NVivo using thematic analysis, guided by the initial programme theory from the realist synthesis. Analysis will remain open to emergent themes that support further theory refinement. Similar to the above integration, quantitative and qualitative results will be compared (producing joint display table), and quantitative and qualitative data will be merged for each case (producing case histories and a mixed method matrix).

Research assistants from the various provinces will co-analyse quantitative, qualitative and mixed methods evidence. They will identify CMO configurations, first within each primary care clinic (case) and then across sites. All team members will be involved in certain steps of the analysis. A recap table ${ }^{76}$ will be constructed using columns to separate components of the initial theory and rows representing different cases. This approach will facilitate within-case analysis, highlighting similarities or discrepancies between data sources. It will also facilitate cross-case analysis to identify patterns (demiregularities or semipredictable patterns) across cases. Analysis of CMO configurations will help complete, confirm or modify the components of our initial theory and ultimately produce a refined theory explaining how and why CM works, in specific contexts, and for specific categories of patients. Results will be reported in line with the Realist And Meta-narrative Evidence Syntheses: Evolving Standards (RAMESES II) reporting standards for realist evaluation. ${ }^{77}$

\section{Objective 3: to identify the next steps towards CM spread in primary care across Canada}

The Technique for Research of Information by Animation of a Group of Experts (TRIAGE) method will be used to reach consensus among all stakeholders about the next steps forward with spread (expansion and extension), in light of our case study results. The process of developing a shared understanding from the different stakeholders' perspectives through discussion improves progress of an innovation towards spread. ${ }^{24}$ TRIAGE is a research method based on the attainment of a group consensus to supply first-hand information for decision making. ${ }^{78}$ It is a structured and inductive method of data collection comprising three successive phases: preparation, individual production and interactive production.

\section{Preparation}

A full-day meeting will be organised, gathering the tripartite structure (clinical, scientific and policy maker leads) of all pan-Canadian SPOR Networks in Primary and Integrated Health Care Innovations (PIHCI) and at least one individual/patient from each province in order to embody categories of stakeholders across Canada. PIHCI is a network building on regional and national achievements in community-based primary and integrated healthcare. ${ }^{79}$ During this preparation phase, a brief executive summary of project results will be produced and tailored to inform each specific audience and category of stakeholders. The evaluation question that will be discussed and disseminated to the participants is as follows: based on your own experience, what should be the next steps towards the spread of CM in primary care in your area of expertise (patient engagement, clinical care, policy and research)? 
Individual production

All stakeholders will receive the executive summary of the results, 2 months prior to the meeting, and will be asked to provide a maximum of five statements in response to the question stated above. Beyond five statements, information is expected to become redundant. ${ }^{78}$ These statements will be kept confidential and sent back to the organisation team.

\section{Interactive production}

This phase will take place during the full-day meeting. The project and results will be presented to all participants. Each group of stakeholders will gather to identify, by consensus, the most important and relevant statements among those brought forth in their stakeholder category. An expert animator will act as a facilitator and lead interactions among group experts. The interactive step of TRIAGE relies on a prominent visual aid. A wall of the room will be used and divided into three main sections: memory, groupings and selection. The memory section is, in fact, a bank of all statements gathered in the previous step, which have been numbered and transcribed. As group interactions occur, the selection process will evolve, with cards moving from one section to another, from left (memory) to right (selection). It will also be possible to modify the statements. 'Selected' statements will also be ranked and prioritised. At the end of the meeting, each group of stakeholders will present their selected statements in order of priority.

\section{Patient and public involvement}

This project was developed in close collaboration with patient-partners, with which we developed a trusty relationship and a collaborative approach. These partners are listed as coauthors (GG, CL, JR, AS, CS, VS, MW). These patient-partners were involved in the elaboration of the research questions that were relevant from their perspectives. Patient-partners will advise on ways to enhance study feasibility and patient's acceptability. They will be engaged in interpretation of data. Results will be disseminated to patients through lay language newsletters and local media.

\section{ETHICS AND DISSEMINATION}

All participants will provide informed consent prior to engagement and recruitment. In addition, certificates of approval will be obtained in each of the provinces before data collection is commenced. If appropriate, adherence to Chapter 9 of the Tri-Council Policy Statement: Ethical Conduct for Research Involving Humans (TCPS2) (2014) will be observed and upheld.

This 4-year multiple-case, mixed method study will result in the potential for great impact with stakeholders but mostly for individuals / patients. New evidence-based knowledge will be provided on the implementation of CM interventions, which can contribute to improve care integration for individuals/patients who frequently use healthcare services and ultimately reduce ineffective healthcare use and costs. The proposed design will allow adapting the knowledge acquired on CM to local contexts, the first essential step towards implementation. ${ }^{80}$ Moreover, recognition of facilitators and barriers to implementation as well as the influence that context exerts on outcomes will pave the way for the spread of CM in primary care settings in different Canadian jurisdictions.

This study built on various strengths but mostly on the engagement of knowledge users who were and will be involved throughout the entire process to ensure that the new knowledge generated by CM in primary care will be refined and tailored to their own specific needs. ${ }^{80}$ These stakeholders will then be best suited to further adapt CM knowledge to their own local context and to increase the chance of successfully implementing CM in their setting. ${ }^{80}$ All of these steps will increase spread and positively influence the healthcare system as well as individuals/patients/communities and clinicians' experiences and outcomes. ${ }^{23} 2435$

This study builds on many important aspects related to the rigour of the approach and methodology. As such, all stakeholders, including individuals/patients, from the five provinces (SK, QC, NB, NS and NL) already working together, have participated in the elaboration of research questions that were relevant from their perspectives. This partnership with stakeholders is strengthened by a solid engagement plan as well as a relevant knowledge transfer plan tailored for each stakeholder audience. The conceptual basis of this study is based on a rigorous research plan that unifies key constructs from published implementation theories (CFIR) ${ }^{35}$ as well as a framework combining the concepts of primary care and integration of care (Valentijn). ${ }^{36}$ The intervention is evidence based and shaped for individuals/patients who frequently use healthcare services. ${ }^{8-12}{ }^{81}$ As for data collection, appropriate sampling strategies will be pursued, while data quality and reliability will be ensured through three main strategies $^{25}$ : the 10 case histories will integrate relevant qualitative and quantitative data in a master database; the database will contain sufficient information about data collection; and data collection will follow published methods. Validity of the study will be ensured by mixing qualitative and quantitative methods (comparison of results and data merging), multiple data sources and evaluators' triangulation. ${ }^{25}$ Transferability will be ensured by several strategies such as theoretical basis, observation replication across cases ${ }^{25}$ and thorough description of the context. ${ }^{60}$

While some challenges are expected with this study, mitigated strategies are nevertheless proposed. To ensure meaningful involvement of all provinces and team members in the project, relationships and team building will be nurtured and stakeholders will be encouraged to speak in their preferred language (English or French). Being engaged with patients as research partners over the last 4 years, solutions have been developed to accommodate their needs, for example, help with a wheelchair, 
being flexible regarding schedule if hospitalisation or deterioration, training. Partnerships will also be monitored annually. The circumstances of this vulnerable clientele may also influence data collection as well as study validity. This challenge will be overcome by research assistants administrating the questionnaire to patients and assisting them as needed. In a similar study conducted by our team, a $93 \%$ retention rate was achieved, demonstrating the efficacy of our strategies. ${ }^{59}$

Based on popular conceptual frameworks and rigorous methodology, design and methods, this pan-Canadian study holds promise to guide policy decision making and to ultimately and positively impact health services systems as well and most importantly the health of Canadians. This study will generate findings on the implementation of CM in primary care for individuals/patients with chronic conditions and complex healthcare needs who frequently use healthcare services, as well as to implement an evidence-based intervention that will improve the care experience and outcomes and will mitigate ineffective use and costs.

\section{Author affiliations}

1Département de Médecine de Famille et Médecine d'urgence, Université de Sherbrooke, Sherbrooke, Quebec, Canada

${ }^{2}$ Département des Sciences de la Santé, Université du Québec à Chicoutimi, Chicoutimi, Quebec, Canada

${ }^{3}$ Primary Healthcare Research Unit, Memorial University, St-John's, Newfoundland and Labrador, Canada

${ }^{4}$ Department of Family Medicine, Dalhousie University, Halifax, Nova Scotia, Canada ${ }^{5}$ Department of Nursing and Health Sciences, University of New Brunswick,

Fredericton, New Brunswick, Canada

${ }^{6}$ Department of Academic Family Medicine, University of Saskatchewan, Saskatoon, Saskatchewan, Canada

${ }^{7}$ Department of Family Medicine, Université McGill, Montréal, Quebec, Canada

${ }^{8}$ School of Social Work, Universite de Sherbrooke, Sherbrooke, Quebec, Canada

${ }^{9}$ Département des sciences de la santé communautaire, Université de Sherbrooke,

Sherbrooke, Quebec, Canada

${ }^{10}$ Faculté de Pharmacie, Université Laval, Québec

${ }^{11}$ Department of Family Medicine and Emergency Medicine, Université Laval,

Quebec, Canada

${ }^{12}$ UETMIS and CRCHUS, CIUSSS de I'Estrie - CHUS, Sherbrooke, Quebec, Canada

${ }^{13}$ School of Nursing, McMaster University, Hamilton, Ontario, Canada

${ }^{14}$ Sturgeon Lake First Nation, Sturgeon Lake, Saskatchewan, Canada

${ }^{15}$ Department of Health and Community Services, St-John's, Newfoundland and

Labrador, Canada

${ }^{16}$ Centre intégré universitaire de santé et de services sociaux du Saguenay-LacSaint-Jean, Chicoutimi, Quebec, Canada

${ }^{17}$ Centre de pédiatrie sociale Sud-Est (CPSSE), Memramcook, New Brunswick, Canada

${ }^{18}$ Nova Scotia Health Authority, Halifax, Nova Scotia, Canada

${ }^{19}$ Horizon Health Network, Miramichi, New Brunswick, Canada

${ }^{20}$ Unité SOUTIEN du Québec, Montréal, Quebec, Canada

${ }^{21}$ Centre intégré universitaire de santé et services sociaux de l'Estrie - Centre

hospitalier universitaire de Sherbrooke, Sherbrooke, Quebec, Canada

${ }^{22}$ Ministere de la Sante et des Services sociaux Quebec, Quebec, Quebec,

Canada

${ }^{23}$ Institut national d'excellence en santé et en services sociaux, Québec, Quebec, Canada

${ }^{24}$ Maritime SPOR SUPPORT Unit, Halifax, Nova Scotia, Canada

${ }^{25}$ NaviCare/SoinsNavi, St-John, New Brunswick, Canada

${ }^{26} \mathrm{NL}$ SPOR SUPPORT Unit, St. John's, Newfoundland and Labrador, Canada

${ }^{27}$ Chronic Disease Prevention and Management Health, Government of New Brunswick, Fredericton, New Brunswick, Canada
Acknowledgements The authors would like to thank the Unité de Recherche Clinique et Épidémiologique (URCE) of the Centre de recherche du CHU de Sherbrooke for their support in coordinating the preparation and revisions of this manuscript, and the Method development platform - Quebec SPOR SUPPORT Unit for their methodological support.

Contributors $\mathrm{CH}, \mathrm{M}-\mathrm{CC}, \mathrm{KA}-\mathrm{B}, \mathrm{FB}, \mathrm{SD}$ and VRR are the principal investigators of the study. All authors contributed to the conception, the design of the study, editing and final approval of the protocol and will either be involved in data collection or interpretation.

Funding This work is supported by the Canadian Institutes of Health Research (CIHR) - Operating Grant: SPOR PIHCI Network: Programmatic Grants (grant number 397896) and other partners such as Axe santé-Population, organisations et pratiques du CRCHUS, Centre de recherche du CHUS, Département de médecine de famille et médecine d'urgence (Université de Sherbrooke), Fondation de L'Université de Sherbrooke, Fondation de Ma Vie, Fonds de recherche du Québec en santé, Institut universitaire de première ligne en santé et services sociaux, Ministère de la santé et des services sociaux du Québec, New Brunswick Health Research Foundation, Nova Scotia Health Authority, Faculty of Medicine of Dalhousie University and Dalhousie Medical Research Foundation, Réseau-1 Québec, Saskatchewan Health Research Foundation and Université de Sherbrooke and Université du Québec à Chicoutimi.

Disclaimer The funders played no role in designing the study, neither did they in data collection, analysis and interpretation or in the writing of the manuscript.

Competing interests None declared.

Patient consent Not required.

Ethics approval This project received approval from the CIUSSS de l'Estrie CHUS Research Ethic Board (project number MP-31-2019-2830).

Provenance and peer review Not commissioned; peer reviewed for ethical and funding approval prior to submission.

Open access This is an open access article distributed in accordance with the Creative Commons Attribution Non Commercial (CC BY-NC 4.0) license, which permits others to distribute, remix, adapt, build upon this work non-commercially, and license their derivative works on different terms, provided the original work is properly cited, appropriate credit is given, any changes made indicated, and the use is non-commercial. See: http://creativecommons.org/licenses/by-nc/4.0/.

\section{REFERENCES}

1. Bodenheimer T, Berry-Millett R. Follow the money--controlling expenditures by improving care for patients needing costly services. N Engl J Med 2009;361:1521-3.

2. Department of Health. Caring for people with long term conditions: An education framework for community matrons and case managers UK: Department of Health - Long term conditions team; 2006. http:// webarchive.nationalarchives.gov.uk/20130107105354/http://www. dh.gov.uk/prod consum dh/groups/dh digitalassets/@dh/@en/ documents/digitalasset/dh_4134012.pdf (accessed 22 Nov 2017).

3. Wodchis W. Health Links and Beyond: The Long and Winding Road to Person-Centred Care; 2013. Ontario, Canada: High cost users: driving value with a patient-centered health system, 2012. www. northwestlhin.on.ca/./ /./nw/./High\%20Cost\%20Users.pdf.

4. Commission de la réforme des services publics de l'Ontario. Des services publics pour la population ontarienne: cap sur la viabilité et l'excellence Ottawa: Gouvernement de I'Ontario; 2012. http://www. fin.gov.on.ca/fr/reformcommission/chapters/report.pdf (accessed 17 Nov 2017).

5. Doupe MB, Palatnick W, Day S, et al. Frequent users of emergency departments: developing standard definitions and defining prominent risk factors. Ann Emerg Med 2012;60:24-32.

6. Sun BC, Burstin HR, Brennan TA. Predictors and outcomes of frequent emergency department users. Acad Emerg Med 2003;10:320-8

7. Zuckerman S, Shen YC. Characteristics of occasional and frequent emergency department users: do insurance coverage and access to care matter? Med Care 2004;42:176-82.

8. Althaus F, Paroz S, Hugli O, et al. Effectiveness of interventions targeting frequent users of emergency departments: a systematic review. Ann Emerg Med 2011;58:41-52.

9. Hudon C, Chouinard MC, Lambert M, et al. Effectiveness of case management interventions for frequent users of healthcare services: a scoping review. BMJ Open 2016;6:e012353. 
10. Kumar GS, Klein R. Effectiveness of case management strategies in reducing emergency department visits in frequent user patient populations: a systematic review. J Emerg Med 2013;44:717-29.

11. Soril LJ, Leggett LE, Lorenzetti DL, et al. Reducing frequent visits to the emergency department: a systematic review of interventions. PLoS One 2015; 10:e0123660.

12. Hudon C, Chouinard MC, Aubrey-Bassler K, et al.Case Management for Frequent Users of Health Care Services With Chronic Diseases in Primary Care: A Systematic Mixed Studies Review.NAPCRG Annual Meeting Montreal (Canada). 2017.

13. Blank FS, Li H, Henneman PL, et al. A descriptive study of heavy emergency department users at an academic emergency department reveals heavy ED users have better access to care than average users. J Emerg Nurs 2005;31:139-44.

14. Brodeur M, Chouinard MC, Hudon C. Frequent Users Experience of Healthcare Services: A Qualitative Metasummary. Montreal (Canada): NAPCRG Annual Meeting, 2017.

15. Hunt KA, Weber EJ, Showstack JA, et al. Characteristics of frequent users of emergency departments. Ann Emerg Med 2006;48:1-8.

16. Ruger JP, Richter CJ, Spitznagel EL, et al. Analysis of costs, length of stay, and utilization of emergency department services by frequent users: implications for health policy. Acad Emerg Med 2004:11:1311-7.

17. Billings $\mathrm{J}$, Raven MC. Dispelling an urban legend: frequent emergency department users have substantial burden of disease. Health Aff 2013;32:2099-108.

18. Grembowski D, Schaefer J, Johnson KE, et al. A conceptual model of the role of complexity in the care of patients with multiple chronic conditions. Med Care 2014;52(Suppl 3):S7-S14.

19. Schoen C, Osborn R, Squires D, et al. New 2011 survey of patients with complex care needs in eleven countries finds that care is often poorly coordinated. Health Aff 2011;30:2437-48.

20. Raven MC, Kushel M, Ko MJ, et al. The effectiveness of emergency department visit reduction programs: a systematic review. Ann Emerg Med 2016;68:467-83.

21. Case Management Society of America. What is a case manager? http://www.cmsa.org/who-we-are/what-is-a-case-manager/ (accessed 19 Oct 2017)

22. Hudon C, Chouinard MC, Lambert M, et al. Key factors of case management interventions for frequent users of healthcare services: a thematic analysis review. BMJ Open 2017;7:e017762.

23. Canadian Institutes of Health Research. The knowledge to action cycle. http://www.cihr-irsc.gc.ca/e/documents/kt in health care chapter 5.1 e.pdf (accessed 19 Oct 2017).

24. Gupta A, Thorpe C, Bhattacharyya O, et al. Promoting development and uptake of health innovations: The Nose to Tail Tool. F1000Res 2016;5:361

25. Yin RC. Case study research: Design and methods. 5th ed. Thousand Oaks, CA: Sage Publications, 2014

26. Gerring J. Case study research: principles and practices. Cambridge: Cambridge University Press, 2007.

27. Wensing M, Grol R. Observational evaluation of implementation strategies. In: Grol R, Wensing M, Eccles M, eds. Improving patient care: The implementation of change in clinical practice. Oxford: Elsevier, 2013:248-55

28. Pluye P, Bengoechea EG, Granikov V, et al. A world of possibilities in mixed methods: review of the combinations of strategies used to integrate qualitative and quantitative phases, results and data. Int $J$ Mult Res Approaches 2018;10:41-56.

29. Levesque JF, Haggerty JL, Hogg W, et al. Barriers and facilitators for primary care reform in canada: results from a deliberative synthesis across five provinces. Healthc Policy 2015;11:44-57.

30. Harris MF, Advocat J, Crabtree BF, et al. Interprofessional teamwork innovations for primary health care practices and practitioners: evidence from a comparison of reform in three countries. $J$ Multidiscip Healthc 2016;9:35-46.

31. Mazurek Melnyk B, Fineout-Overholt E. Evidence-based practice in nursing \& healthcare: A guide to best practice. 3 edn. Philadelphia, PA: Wolters Kluwer, 2015

32. Eisenhardt KM. Building theories from case study research. Acad Manage Rev 1989;14:532-50.

33. Fitzgerald L, Dopson S. Comparative case study designs: their utility and development in organisational research. In: Buchanan D, Bryman A, eds. The SAGE Handbook of Organizational Research Methods. Los Angeles, London: SAGE, 2009.

34. Champagne F, Brousselle A, Hartz Z, et al. L'analyse de l'implantation. In: Brousselle A, Champagne F, Contandriopoulos A, eds. L'évaluation: Concepts et méthodes. Montréal: Presses de I'Université de Montréal, 2011:237-73.

35. Damschroder LJ, Aron DC, Keith RE, et al. Fostering implementation of health services research findings into practice: a consolidated framework for advancing implementation science. Implement Sci 2009;4:50.

36. Valentijn PP, Schepman SM, Opheij W, et al. Understanding integrated care: a comprehensive conceptual framework based on the integrative functions of primary care. Int $J$ Integr Care 2013;13:e010.

37. Haith MP, Whittingham KA. How to use action learning sets to support nurses. Nurs Times 2012;108:12-14.

38. Ross S, Curry N, Goodwin N. Case Management: What it is and how it can best be implemented London. UK: The King's Funds, 2011. Available from. https://www.kingsfund.org.uk/sites/default/files/ Case-Management-paper-The-Kings-Fund-Paper-November-2011 0.pdf. (accessed 22 Nov 2017).

39. Challis D, Hughes J, Berzins K, et al. Self care and case management in long term conditions: the effective management of critical interfaces London. UK: University of Manchester, 2010. http://www.pssru.ac.uk/pub/MCpdfs/SCCMfr.pdf. (accessed 22 Nov 2017).

40. Freund T, Peters-Klimm F, Rochon J, et al. Primary care practicebased care management for chronically ill patients (PraCMan): study protocol for a cluster randomized controlled trial [ISRCTN56104508]. Trials 2011;12:163.

41. Barlow J, Wright C, Sheasby J, et al. Self-management approaches for people with chronic conditions: a review. Patient Educ Couns 2002:48:177-87.

42. Krieg C, Hudon C, Chouinard MC, et al. Individual predictors of frequent emergency department use: a scoping review. BMC Health Serv Res 2016:16:594.

43. Candian Institutes of Health Research, 2017. Dynamic cohort of complex, high system users - 2011-2015: governement of canada http://www.cihr-irsc.gc.ca/e/50129.html\#section_3_4_5 (accessed 10 Oct 2017).

44. Boehlen FH, Joos A, Bergmann F, et al. [Evaluation of the German Version of the "INTERMED-Self-Assessment"-Questionnaire (IM-SA) to Assess Case Complexity]. Psychother Psychosom Med Psychol 2016:66:180-6.

45. National Case Management Network of Canada, Canadian Standards of Practice in Case Management. Connect, collaborate and communicate the power of case management Ottawa 2009. http://www.ncmn.ca/resources/documents/english\%20standards\% 20for\%20web.pdf (accessed 19 Oct 2016).

46. National Collaboration for Integrated Care and Support. Integrated care and support: Our shared commitment. London, 2013. https:// www.gov.uk/government/uploads/system/uploads/attachment_data/ file/198748/DEFINITIVE_FINAL_VERSION_Integrated_Care_and Support_-_Our_Shared_Commitment_2013-05-13.pdf (accessed 22 Nov 2017)

47. Fortin MF. Fondements et étapes du processus de recherche. Chenelière 2010.

48. MacQuarrie C, saturation T. In: Mills AJ, Eurepos G, Wiebe E, Encyclopedia of case study research. Thousand Oaks, CA: Sage, 2010:927-9.

49. Chew LD, Bradley KA, Boyko EJ. Brief questions to identify patients with inadequate health literacy. Fam Med 2004;36:588-94

50. Hudon E, Hudon C, Couture EM, et al. Validation of a frenchlanguage version of a three-item questionnaire. North American Primary Care Research Group Annual Meeting 2016.

51. Bayliss EA, Ellis JL, Steiner JF. Subjective assessments of comorbidity correlate with quality of life health outcomes: initial validation of a comorbidity assessment instrument. Health Qual Life Outcomes 2005;3:51.

52. Poitras ME, Fortin M, Hudon $\mathrm{C}$, et al. Validation of the disease burden morbidity assessment by self-report in a French-speaking population. BMC Health Serv Res 2012;12:35.

53. Hudon E, Hudon $\mathrm{C}$, Lambert $\mathrm{M}$, et al. Validation of a Frenchlanguage version of a patient-reported measure of integrated care. North American Primary Care Research Group Annual Meeting 2016.

54. Smith $D$, Harvey $P$, Lawn $S$, et al. Measuring chronic condition selfmanagement in an Australian community: factor structure of the revised Partners in Health (PIH) scale. Qual Life Res 2017;26:149-59.

55. Hudon $\mathrm{E}$, Hudon $\mathrm{C}$, Lambert M, et al. Measuring self-management of patient with chronic disease in primary care: Validation of a Frenchlanguage version of the Partner in Health Scale. North American Primary Care Research Group Annual Meeting 2016.

56. Ware JE. User manuel for the SF-36v2 Health survey. 2 edn. Lincoln, RI: Quality Metric Incorporated, 2007.

57. Canadian Institute for Health Information. Patient cost estimator. https://www.cihi.ca/en/spending-and-health-workforce/spending/ patient-cost-estimator (accessed 19 Oct 2017).

58. Dumas JE, Lynch AM, Laughlin JE, et al. Promoting intervention fidelity. Conceptual issues, methods, and preliminary results from 
the EARLY ALLIANCE prevention trial. Am J Prev Med 2001;20(1 Suppl):38-47.

59. Hudon C, Chouinard MC, Dubois MF, et al. Case management in primary care for frequent users of health care services: a mixed methods Study. Ann Fam Med 2018;16:232-9.

60. Miles MB, Huberman AM, Saldaña J. Qualitative Data Analysis: A methods sourcebook. 3edn. London: Sage Publications Inc, 2014.

61. Ramsey S, Willke R, Briggs A, et al. Good research practices for cost-effectiveness analysis alongside clinical trials: the ISPOR RCTCEA Task Force report. Value Health 2005;8:521-33.

62. Fenwick E, O'Brien BJ, Briggs A. Cost-effectiveness acceptability curves--facts, fallacies and frequently asked questions. Health Econ 2004:13:405-15.

63. Craig P, Dieppe P, Macintyre S, et al. Developing and evaluating complex interventions: the new Medical Research Council guidance. BMJ 2008;337:a1655.

64. Pawson R, Tilley N. Realistic evaluation London: Sage, 1997. https:// us.sagepub.com/en-us/nam/realistic-evaluation/book205276 (accessed 22 Nov 2017).

65. Marchal $\mathrm{B}$, van Belle $\mathrm{S}$, van Olmen J, et al. Is realist evaluation keeping its promise? A review of published empirical studies in the field of health systems research. Evaluation. Realistic evaluation. London: Sage Publications, 2012:192-212.

66. Pawson R. The science of evaluation: A realist manifesto. Thousand Oaks, CA: SAGE Publications, 2013.

67. Greenhalgh T, Humphrey C, Hughes J, et al. How do you modernize a health service? a realist evaluation of whole-scale transformation in London. Milbank Q 2009;87:391-416.

68. Rycroft-Malone J, Fontenla M, Bick D, et al. A realistic evaluation: the case of protocol-based care. Implement Sci 2010;5:38.

69. Redfern S, Christian S, Norman I. Evaluating change in health care practice: lessons from three studies. J Eval Clin Pract 2003;9:239-49.
70. Kennedy A, Rogers A, Gately C. Assessing the introduction of the expert patients programme into the NHS: a realistic evaluation of recruitment to a national lay-led self-care initiative. Primary Health Care Research and Development 2005:6:137-48.

71. Jeffs L, Jain AK, Man RH, et al. Exploring the utility and scalability of a telehomecare intervention for patients with chronic kidney disease undergoing peritoneal dialysis - a study protocol. BMC Nephrol 2017; 18:155.

72. Tremblay D, Touati N, Roberge D, et al. Conditions for production of interdisciplinary teamwork outcomes in oncology teams: protocol for a realist evaluation. Implement Sci 2014;9:76.

73. Hudon C, Chouinard M-C, Aubrey-Bassler K, et al. Case management in primary care among frequent users of healthcare services with chronic conditions: protocol of a realist synthesis. BMJ Open 2017;7:e017701.

74. Manzano A. The craft of interviewing in realist evaluation. Sage Journals 2016;22:342-60.

75. Pawson R. Theorizing the interview. Br J Sociol 1996:47:295-314.

76. O'Cathain A, Murphy E, Nicholl J. Three techniques for integrating data in mixed methods studies. BMJ 2010;341:c4587.

77. Wong G, Westhorp G, Manzano A, et al. RAMESES II reporting standards for realist evaluations. BMC Med 2016;14:96.

78. Gervais $M$, Pépin G. Triage: A new group technique gaining recognition in evaluation. Evaluation Journal of Australasia 2002;2:45-9.

79. ClHR home page. (PIHCl) P-CSNiPalHCl.

80. Castiglione SA, Ritchie JA. Moving into action: We know what practices we want to change, now what? An implementation guide for health care practitioners, 2012.

81. Hudon C, Chouinard MC, Diadiou F, et al. Case management in primary care for frequent users of health care services with chronic diseases: a qualitative study of patient and family experience. Ann Fam Med 2015;13:523-8. 